\title{
Alternative Numerical Validation Methodology for Short-Term Development Projects
}

\author{
Mohamed P.Hassan ${ }^{1,}$, Abdullah S. Mahmud ${ }^{1, b}$, A.S. Mohd Rafie ${ }^{1, c}$ \\ and Rizal Zahari, ${ }^{1, d}$ \\ ${ }^{1}$ Aerospace Engineering, Faculty of Engineering, Universiti Putra Malaysia, 43400 Serdang, \\ Selangor Darul Ehsan, Malaysia \\ amohamedhph@gmail.com, basmahmud@hotmail.com, 'shakrine@eng.upm.edu.my, \\ drizal@eng.upm.edu.my
}

Keywords: Numerical, validation, airframe, structure, innovation, FEA.

\begin{abstract}
Virtual prototyping has been increasingly taking over the process of sole physical tests. Companies are reporting up to $80 \%$ reduction in errors when using virtual tests through the design process. Conventional numerical validation methodology however, is not as beneficial for shortterm projects because any new numerical scenario has to be validated before being used. Although during the conceptual stage, relative values can be sufficient. The alternative methodology proposed also uses realistic loads. It comprise applying these loads on a functioning structure to verify them. The modified version of the structure is then relatively validated by being tested under these verified loads. Thus, bypassing the physical tests requirement. Aerodynamic loads are acquired from simulating the Gulfstream IV-SP forward fuselage during climbing, cruising and landing. Mechanical loads are acquired from estimating structural weight and impact load during landing. In total, three finite element models were created. Autodesk softwares were used to perform CFD and FEA. Only greater loads were applied during FEA. Results simplified neglected cruising data for having lowest values. Comparing estimated weights of functional and modified structures showed a possible $15 \%$ weight savings. While the FEA results showed a promising $45 \%$ less inquired stress within the modified structure.
\end{abstract}

\section{Introduction}

The virtual prototype is a digital simulation of the respective physical prototype. It is increasingly used for product verification, to verify product throughout its whole lifecycle. Many aerospace companies have already increased their use of virtual prototyping. For example, design cycle reductions of $60 \%$ in a project was achieved in Chengdu Aircraft, with software applied for conceptual, detailed and engineering design [1]. The use of digital mock-up (DMU) system i.e. virtual prototyping, Boeing reduced errors and reworks by $70-80 \%$, equivalent to about 100,000 design hours.

Normally, the validation methodology of a general numerical analysis scenario is conducted using parallel physical tests for verification. Then similar numerical analysis scenarios are applied to variant cases, without requiring additional parallel physical tests. An example of this conventional validation methodology is discussed in reference [2], S. Georgiadis et al. validated a numerical analysis scenario by parallel physical tests. The scenario simulates "Bird-strike" on "Boeing 787 composite moveable trailing edge". S. Georgiadis et al. then proved the numerical scenario could substitute further conventional certification physical tests, once it have been validated with parallel physical tests.

\section{Methodology}

Conventional validation methodology is helpful for long-term projects. Although short-term projects based on numerical analysis would not be able to apply such methodology, because it is more beneficial to conduct physical tests only. That is, results acquired from the conducted physical tests are more established than these obtained from the verified numerical analysis scenarios. Hence, for short-term projects, numerically verifying all the numerical analysis scenarios before 
their application is less time consuming than verifying a similar number of general numerical analysis scenarios using parallel physical tests. Thus, an alternative validation methodology is established to allow faster development and optimization. Hence eliminating the need for tests on a physical prototype within the conceptual stage.

The proposed alternative validation methodology, shown in Fig. 1, where the numerical analysis scenario is applied on a functional conventional structure. The scenario uses realistic acquired loads. If Finite Element Model (FEM) of the conventional structure passes "stress resistance test", the numerical analysis scenario is verified. The structure pass the test, when the maximum enquired stress during the scenario application is less than allowable material stress (yield stress) and produces a Factor of Safety (FOS) equal to 1.5 or more. The exact same verified scenario can then be used to validate a modified version of the original conventional structure. The "modified structure" has to have relatively similar dimensions and to be capable of experiencing same analysis scenario e.g. same load application areas/points, thus allowing the scenario to function similarly. The validation process is then concluded based on the stress resistance test, in a similar manner to that applied to verify the scenario using the conventional structure. Since this case study structure is an airframe and its weight is sensitive, the modified structure weight have to be equal to or less than the respective conventional structure weight, to provide an added value. Thus, a weight comparison step have to precede the application of the verified scenario on the modified structure.

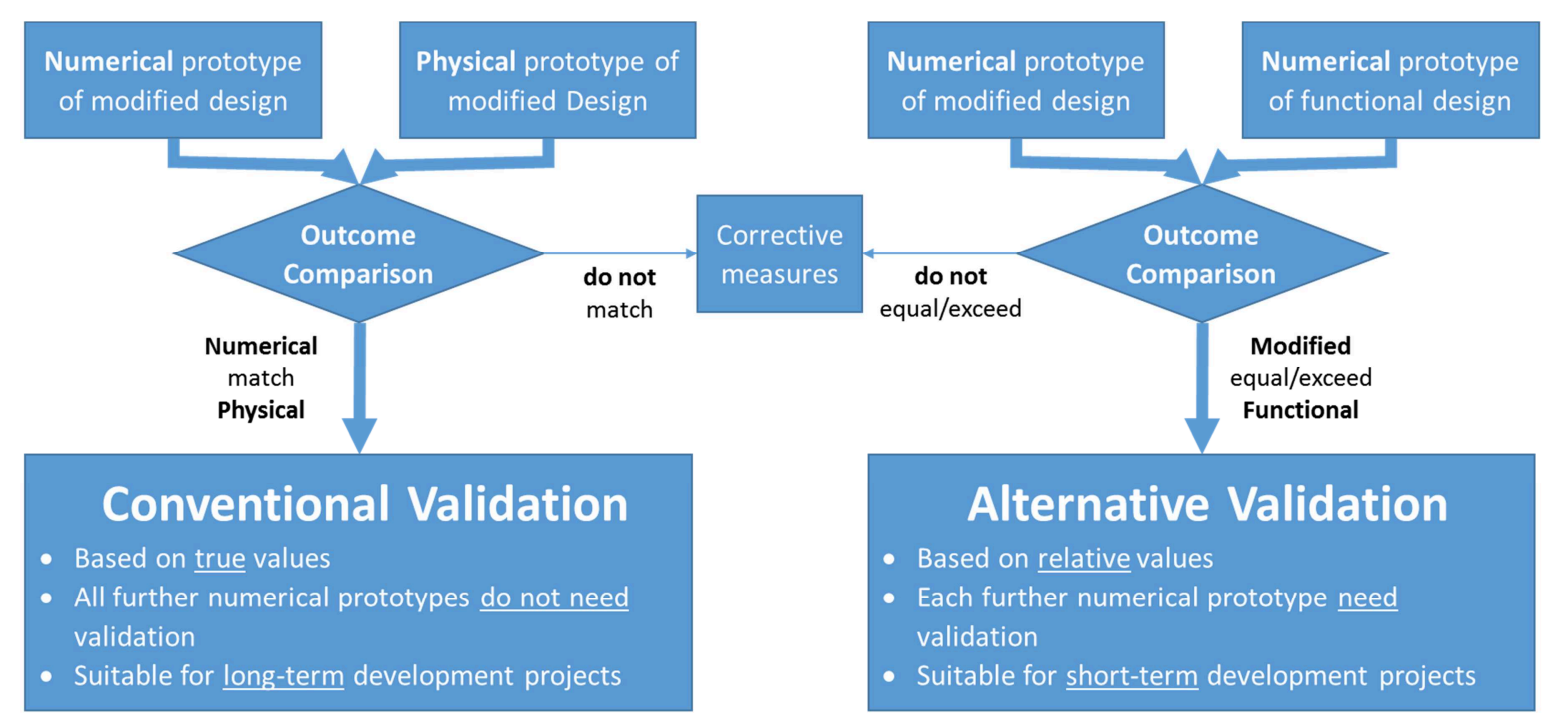

Fig. 1. Validation methodologies layout.

The loads used in the paper's case study scenario are acquired from simulating carrying structure in the Computational Fluid Dynamics (CFD) software. The carrying structure in the case study is the Gulfstream IV-SP forward fuselage. The acquired loads are then verified by applying them on the conventional structure, which should be able to withstand them in addition to special loads i.e. landing impact on forward gear. Failure to do so, would point out an error in proposed simulation scenarios. Hence, these scenarios have to be revised accordingly. In case acquired loads are successfully verified, the next step is to apply the same scenario to the new structure to-bevalidated, thus concluding the validation process.

Three models are hence created:

1. Carrying body, to allow acquiring aerodynamic loads on forward fuselage

2. Conventional structure, to verify loads acquired from CFD software

3. The new structure, to be validated using verified loads

Three softwares from Autodesk ${ }^{\circledR}$ are used to accomplish the process:

1. AutoCAD 2014 (Computer Aided Design (CAD) software) to create the required FEMs

2. Simulation CFD 2013 (CFD software) to acquire aerodynamic loads on forward fuselage's FEM 
3. Simulation Multiphysics 2013 (Finite Element Analysis (FEA) software) to facilitate applying aerodynamic and other loads to the conventional structure's FEM for these loads verification and to the new structure's FEM for its design validation

\section{Modeling FEMs}

First, the section of interest was selected and interference with features that might complicate the analysis was avoided. Thus, section was determined to be $500 \mathrm{~mm}$ thick, located at $5.73 \mathrm{~m}$ and extending to $6.23 \mathrm{~m}$ from rear gear centerline, based on detailed diagram acquired from reference [3]. The forward fuselage (Carrying body) FEM was created, based on dimensions presented in references $[3,4]$. That is to be used later within CFD to acquire aerodynamic loads at section of interest. The section presented as two red successive peripheral circles in Fig. 3.

The conventional structure is a transverse section of the carrying body, a cylindrical section of the forward fuselage. The outer diameter of the section is thus the same as the Gulfstream IV-SP fuselage which is $2385 \mathrm{~mm}$. While other internal features specifications were acquired with help of references [3-8]. The conventional structure relative 2D outline and detailed 3D FEM, are shown in Fig. 2.
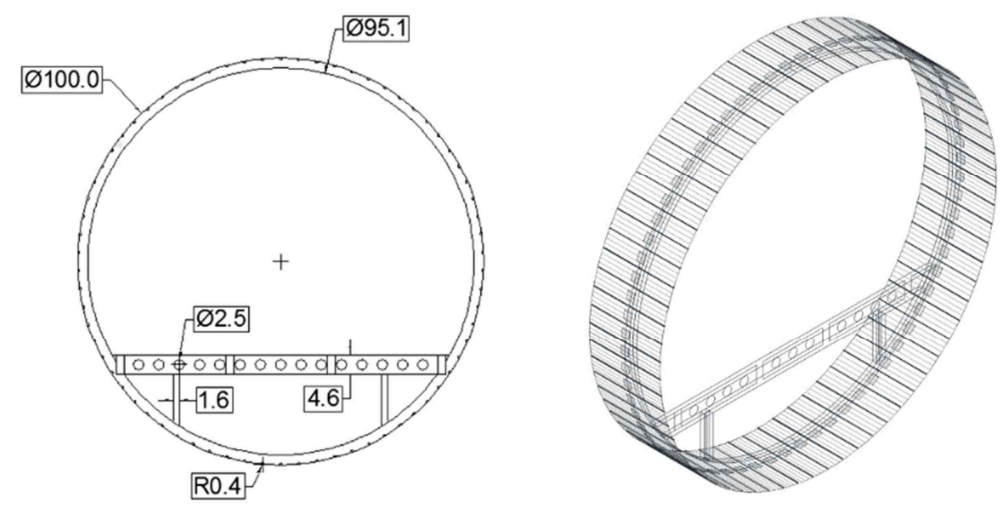

Figure 2. Simplified 2D CAD (left) and 3D CAD (right).

As for the new structure, the dimensions and model features of its FEM are scaled to meet the same outer diameter value of the conventional structure. The case study new structure is based on a novel structure introduced in a recent research [9].

\section{Acquiring loads}

The modeled forward fuselage is imported into the CFD software, where the mesh of predetermined cylindrical section, is selected to acquire loads on it within different possible scenarios i.e. climbing, cruising and landing. The section is shown in Fig. 3, during start of CFD analysis.

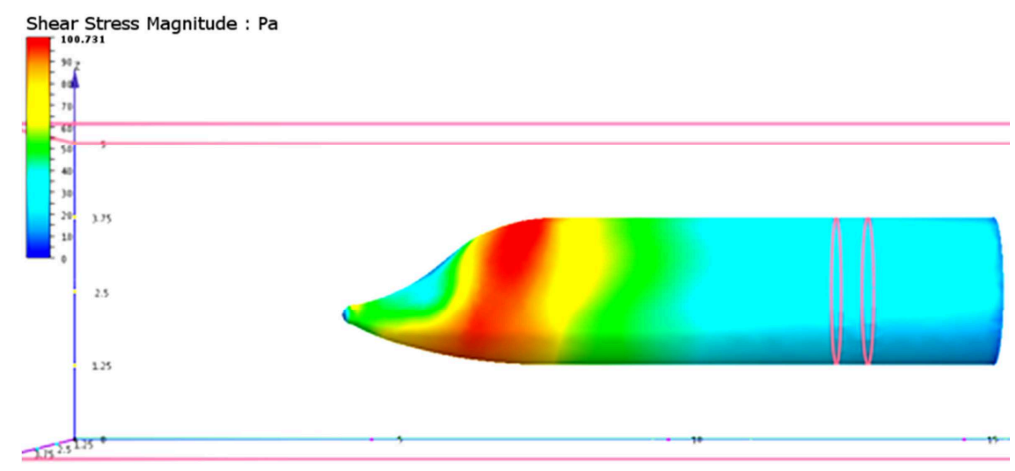

Figure 3. Forward fuselage cruising start (shear stress). 
The climbing scenario was setup, so as the speed of incoming perpendicular airstream, onto model nose, is $850 \mathrm{~km} / \mathrm{h}$ (average cruising speed). The climbing rate was indicated as $4122 \mathrm{ft} / \mathrm{min}$, in the pre-analysis setup. The climbing was simulated by another airstream in a mutual perpendicular direction from above the model. In real takeoff scenario, aircraft would induce an angle of attack so as the resultant airstream is almost perpendicular to aircraft nose, resulting in lower loads. However since this is a validating methodology, extreme conditions where applied within CFD to obtain maximum possible loads. The cruising scenario analysis, shown in Fig. 3, was based on the reference aircraft's maximum speed, which is $935 \mathrm{~km} / \mathrm{h}$. The landing scenario was simulated by applying the same climbing configuration, the climbing rate inverted. Although the specific actual descend rate would have been more accurate to use, the actual value could not be acquire. Both Metric and English units were used to preserve specification as stated in references [3, 10, 11]. The model was placed in a virtual wind tunnel, which have two inlets and two outlets. Each inlet had an opposite outlet. In case of cruising scenario, only one pair of inlet and outlet was used, assuming flight at maximum speed with no deviations. While in case of climbing and landing, the second inlet was used to apply climbing/descend rate.

Structures' weight loads were neglected for being 0.1 of the obtained aerodynamic loads (from CFD). However, the landing impact load was included and is presented by Eq. (1). In the equation, $W$ is aircraft weight at landing and roughly equals $29937.1 \mathrm{~kg}, h_{c g}$ is the height of aircraft's center of gravity (CG) from the static ground line and $l_{m} \& l_{n}$ are the distances measured from the aircraft CG to the main and nose (forward) gear, respectively. The thrust can be added to the equation to determine the maximum load possible, if reverse thrust was not applied $[11,12]$.

$$
\mathrm{F}_{\mathrm{n}}=W\left[\left(l_{m}+0.45 h_{c g}\right) /\left(l_{m}+l_{n}\right)\right]+(7 \% \text { Safety Factor })
$$

CG was determined, hence $l_{m}=0.47 \mathrm{~m}, l_{n}=11.17 \mathrm{~m}$ and $h_{c g}=3.16 \mathrm{~m}$. The CG location was verified by rough visually comparison with CG locations of similar aircrafts, in reference [13].

\section{Loads verification}

In order to verify the consistency of loads, the FEM of the conventional structure was imported to the FEA software. Edges on both sides where constrained, using a peripheral transition fixation. The FEM inter-contacts/joints, were assumed welded, where the study is more concerned with the whole design inter-arrangement. Analysis type was "Static Stress with Linear Material Models". FEM for conventional structure was simulated for climbing and landing scenarios. The cruising scenario was omitted, as analysis is concerned with the largest acting loads, according to loads acquired from CFD.

$$
\text { FOS = Material Strength / Allowed Design Load }
$$

Aircraft structural factor of safety (FOS) common value of 1.5 was used [14]. Where in Eq. (2) material strength is the yield strength [15]. Thus if the resultant stresses from the FEA software achieve a FOS equal to 1.5 or higher, the loads are verified.

\section{Validation process}

As explained earlier, the first step is to confirm, the weight of the modified structure is less or equal to that of the conventional structure. The second step is then conducted by applying previously verified loads and constrains to modified structure FEM, in the same manner used for conventional structure FEM.

The validation is concluded by comparing results from FEA for modified structure with those of the conventional. It the modified structure experienced less or equal stresses to these experienced by the respective conventional structure, the new modified structure is rendered as an added value. 


\section{Results and Discussion}

The different flight stages have been simulated, as discussed earlier, within different CFD analysis scenarios, using the forward fuselage FEM. Three analysis scenarios were applied: climbing, cruising and landing. For each scenario, a set of data was generated. The data sheets were then simplified and forces data plotted as shown in Fig. 4. The largest load resultants were realized to be during climbing and landing stages, while during cruising loads were minimal and almost constant. Thus, only values for resultant force along Z-axis (FZ) and resultant moment around Xaxis (MX) were used.

The maximum values during climbing and landing for $\{\mathrm{FZ}, \mathrm{MX}\}$, were $\{-3467.12,2103.37\}$ (Newton) and $\{9535.09,-5785.28\}$ (Newton / meter), respectively. Although similar values for torque were acquired $\{-740.17,1423.18\}$ (Newton / meter), it was considered relatively small and was omitted from FEA, to avoid further complexity. From CFD analysis, location of resultant forces acting centers was determined, as well as, the aerodynamic loading area on the section, however both were neglected, to avoid further complexity.

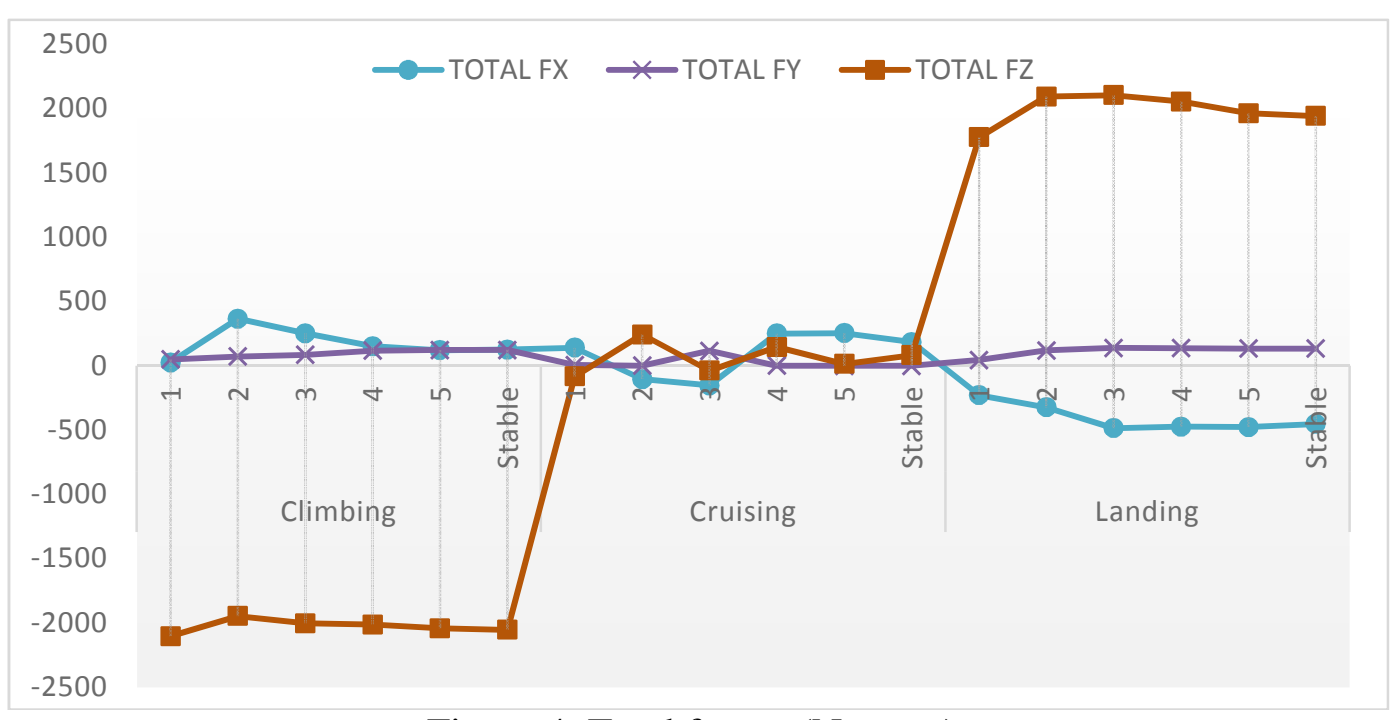

Figure 4. Total forces (Newton).

The conventional structure was used to verify loads (scenarios). Its weight was estimated to be $18.5 \mathrm{~kg}$. Thus, by applying Eq. (1), we obtained a moment at the section, arising from impact load at forward gear, equal to 25083.83N.m. Then by applying the FEA scenario for the conventional structure the maximum inquired stresses during climbing and landing were $126.9 \mathrm{MPa}$ and $76.96 \mathrm{MPa}$, respectively. The loads/scenario verification was then concluded by using Eq. (2), which rendered FOS higher than 1.5, giving values of 2.55 and 4.21 for climbing and landing respectively.

Similar to the conventional structure, the new modified structure weight was determined before the analysis and was estimated to equal $16.19 \mathrm{~kg}$. The verified loads/scenario was applied to the modified structure, rendering maximum inquired stress during climbing and landing equal to $69.49 \mathrm{MPa}$ and $42.16 \mathrm{MPa}$, respectively. Which gave FOS values of 4.66 and 7.69 , respectively. Hence the structure was considered valid. The case study modified structure and based on this methodology and its relative values comparison provided $15.2 \%$ weight saving and a consistent $45.2 \%$ less inquired stress, over the conventional structure.

\section{Summary}

New given airframe structures can be validated numerically without requiring a physical prototype being tested in parallel. This validation methodology takes place in four steps. First, the conventional structure is modeled as close to the currently operating structure as possible. Second, loads affecting the carrying body e.g. fuselage, are acquired. These loads are then verified by applying them onto the conventional structure and finally using these loads to validate the new structure. 
The case study new structure used to present the methodology was proved to provide a consistent $45.2 \%$ extra stress resistance during different operation states, while achieving $15.2 \%$ weight saving in its structural parts. Such proof of concept for the case study new structure was achieved using a standalone numerical approach, while shifting the need for a physical prototype test to later design stages. Thus the presented methodology proved to be time saving within the conceptual stage.

The methodology presented within this paper can be further verified using parallel physical prototype testing. That is to prove the presented methodology itself, rather than validating a specific FEM. It is also to verify its applicability to other kinds of structures. Furthermore, the future design methodology is expected to increasingly shift towards virtual practices. Especially considering the amount of development recently achieved within the FEA software capabilities.

\section{Acknowledgements}

We would like to thank M.R. Ajir and Fairuz I. Romli for sharing their ideas about dimensions and loads acquirement. We would also like to thank Autodesk, Inc. and Universiti Putra Malaysia for providing services and facilities, which helped accomplish this paper.

\section{References}

[1] V. P. McConnell, REINFORCED plastics (2007), pp. 24-28.

[2] S. Georgiadis, A. J. Gunnion, R. S. Thomson and B. K. Cartwright, Composite Structures, Vol. 86 (2008), p. 258-268.

[3] Information on http://www.jetsearch.com/index.php/Current-Inventory/1996-gulfstream-g-ivsp-1292.html.

[4] Information on http://www.aoml.noaa.gov/hrd/graphics/N49RF_2.gif.

[5] A. J. Best, Artist, Airbus A320-111 Aircraft Pictures, Airliners.net (2007).

[6] Asiir, Artist, Airbus A300 cross section. [Art]. Wikipedia, the free encyclopedia (2002).

[7] A. Tipster, Artist, B-24 stringers. [Art]. Shorpy.com (2009).

[8] D. Nunez, Artist, Boeing 747 fuselage structure. [Art]. Yahoo! Inc. (2010).

[9] M. P.Hassan and A. S. Mohd Rafie, International Journal of Engineering Research \& Technology (IJERT), Vol. 2 (8) (2013), p. 1611-1615.

[10] Information on http://www.airliners.net/aircraft-data/stats.main?id=238.

[11] Information on http://jetsales.com/comp/types/jets/bgulfstreamIVsp.html.

[12] S. T. Chai and W. H. Mason, in National Technical Information Service, Washington (1997).

[13] J. Roskam, in Airplane Design - Part V: Component Weight Estimation, Lawrence, DAR corporation (2003), p. 117-120.

[14] G. N. Mangurian, in The Aircraft Structural Factor of Safety, Advisory Group for Aeronautical Research and Development (1957), Paris.

[15] D. G. Ullman, The Factor Of Safety As A Design Variable (Appendix C), in The Mechanical Design process (Second Edition), New York, The McGraw-Hill Companies, Inc., 1992, p. 314. 